\title{
PARTITIONS INTO SPECIFIED PARTS WHICH APPEAR A SPECIFIED NUMBER OF TIMES
}

\author{
ELMO MOORE
}

ABSTRACT. Restrictions of this type, which are known to produce partition identities when any natural number may be used as a part, are shown to produce partition identities when only certain parts may be used.

1. Introduction. Let $N$ be the set of natural numbers and let $A, B$, and $S$ be subsets of $N$. $S(n)$ is the number of partitions of $n$ into parts which are elements of $S . A^{*}(n, B)$ is the number of partitions of $n$ into parts which are elements of $B$, such that each part appears exactly $m$ times for some $m \in A$. For example, if $1,2 \in A$ and $3,4,5 \in B$, then $5+4+3+3$ is a partition of 15 of the type enumerated by $A *(15, B)$.

$A$ is admissible applied to $B$ if there exists an $S$ such that $A *(n, B)$ $=S(n)$ for every $n \in N$. In this case, $S$ corresponds to $A$. For example, previous results by this author [6] state that $A$ is admissible applied to $N$ if it is one of the following, where $k, d \in N$ :

$A=\{m \mid m<(2 d-1) k$ and $m \neq(2 i-1) k+j$ with $1 \leq i \leq d-1,0 \leq j \leq$ $k-1\}$, or

$$
\begin{aligned}
& A=\{m \mid m \neq(2 i-1) k+j \text { with } 1 \leq i \leq d, 0 \leq j \leq k-1\}, \text { or } \\
& A=\{m \mid m \neq(2 i-1) k+j \text { with } 1 \leq i, 0 \leq j \leq k-1\} .
\end{aligned}
$$

The purpose of this paper is to show that these sets are admissible applied to $B$ for $B \neq N$. In particular, for each set $A$, there is a class of sets such that $A$ is admissible applied to $B$ for every $B$ in this class. In each case, $N$ is a member of the class and, thus, these results generalize the earlier ones.

\section{Results.}

Theorem 1. Let $k, d \in N, B \subseteq N$ such that

$$
k B \cap 2 d k B \subseteq B \cap 2 k B \subseteq k B \cup 2 d k B \subseteq B \cup 2 k B .
$$

Then the number of partitions of $n$ into elements of $B$, such that no part appears exactly $(2 i-1) k+j$ times with $1 \leq i \leq d-1,0 \leq j \leq k-1$ or more than $(2 d-1) k-1$ times, is equal to the number of partitions of $n$ into elements of

Received by the editors August 16, 1974.

AMS (MOS) subject classifications (1970). Primary 10A45; Secondary $10 \mathrm{~J} 20$.

Key words and phrases. Partitions, generating functions. 


$$
S=[(B \cup 2 k B)-(k B \cup 2 d k B)] \cup[(B \cap 2 k B)-(k B \cap 2 d k B)] .
$$

A result of Glaisher [4], states that for $k \in N$, the set $A=\{i\}_{i=1}^{k-1}$ is admissible applied to $N$. Letting $d=1$ in Theorem 1 , we see that this set is admissible applied to $B$ for every $B$ such that $k B \subseteq B$.

Corollary 1 (Subbarao). Let $k \in N, B \subseteq N$ such that $k B \subseteq B$. Then the number of partitions of $n$ into elements of $B$, such that parts appear at most $k-1$ times, is equal to the number of partitions of $n$ into elements of $S=$ $B-k B$.

Letting $k=1$ in Theorem 1 , we obtain a similar result.

Corollary 2. Let $d \in N, B \subseteq N$ such that $d B \subseteq B$. Then the number of partitions of $n$ into elements of $B$, such that parts appear exactly $2 i$ times with $1 \leq i \leq d-1$, is equal to the number of partitions of $n$ into elements of $S=2[B-d B]$.

When $B=\left\{i d^{j} \mid j \geq 0,1 \leq i \leq d-1\right\}$ in Corollary $2, A$ is not only admissible applied to $B$, but the corresponding set is $A$ itself.

Corollary 3. Let $d \in N, B=\left\{i d^{j} \mid j \geq 0,1 \leq i \leq d-1\right\}$. Then the number of partitions of $n$ into elements of $B$, such that each part appears exactly $2 i$ times with $1 \leq i \leq d-1$, is equal to the number of partitions of $n$ into parts of the form $2 i$ with $1 \leq i \leq d-1$.

Theorem 2. Let $k, d \in N, B \subseteq N$ such that

$$
\begin{aligned}
B & \cap 2 k B \cap(2 d+1) k B \subseteq k B \cap(4 d+2) k B \subseteq(B \cap 2 k B) \\
& \cup(B \cap(2 d+1) k B) \cup(2 k B \cap(2 d+1) k B) \subseteq k B \\
& \cup(4 d+2) k B \subseteq B \cup 2 k B \cup(2 d+1) k B .
\end{aligned}
$$

Then the number of partitions of $n$ into elements of $B$, such that no part appears exactly $(2 i-1) k+j$ times with $1 \leq i \leq d, 0 \leq j \leq k-1$, is equal to the number of partitions of $n$ into elements of

$$
\begin{aligned}
S= & {[(B \cup 2 k B \cup(2 d+1) k B)-(k B \cup(4 d+2) k B)] } \\
& \cup[((B \cap 2 k B) \cup(B \cap(2 d+1) k B) \cup(2 k B \cap(2 d+1) k B)) \\
& \cup[B \cap 2 k B \cap(2 d+1) k B] .
\end{aligned}
$$

Corollary 4. Let $d, u \in N, B=\left\{2^{i} \mid i \geq u-1\right\}$. Then the number of partitions of $n$ into elements of $B$, where no part appears exactly $2 i-1$ times with $1 \leq i \leq d$, is equal to the number of partitions of $n$ into elements of $S=\left\{2^{i} \mid i \geq u\right\} \cup\left\{(2 d+1) 2^{u-1}\right\}$. 
Theorem 3. Let $k \in N, B \subseteq N$ such that $B \cap 2 k B \subseteq k B \subseteq B \cup 2 k B$. Then the number of partitions of $n$ into elements of $B$, where no part appears exactly $(2 i-1) k+j$ times with $1 \leq i, 0 \leq j \leq k-1$, is equal to the number of partitions of $n$ into elements of $S=[(B \cup 2 k B)-k B] \cup[B \cap 2 k B]$.

In conclusion, we note that, for all $k, d, m \in N$, the set $B=\{i m\}_{i=1}^{\infty}$ satisfies the requirements of the three theorems of this paper.

\section{Proofs.}

Proof of Theorem 1. The generating function for $A^{*}(n, B)$ is

$$
\begin{aligned}
\prod_{n \in B}\left[\frac{1}{1-x^{n}}\right. & \left.-\sum_{i=1}^{d-1} \sum_{j=0}^{k-1} x^{((2 i-1) k+j) n}-\sum_{j=(2 d-1) k}^{\infty} x^{j n}\right] \\
& =\prod_{n \in B}\left[\frac{1}{1-x^{n}}-\left(\sum_{i=1}^{d-1} x^{(2 i-1) k n}\right)\left(\sum_{j=0}^{k-1} x^{j n}\right)-\sum_{j=(2 d-1) k}^{\infty} x^{j n}\right] \\
& =\prod_{n \in B}\left[\frac{1}{1-x^{n}}-x^{k n}\left(\frac{1-x^{(2 d-2) k n}}{1-x^{2 k n}}\right)\left(\frac{1-x^{k n}}{1-x^{n}}\right)-\frac{x^{(2 d-1) k n}}{1-x^{n}}\right] \\
& =\prod_{n \in B}\left(\frac{1}{1-x^{n}}\right)\left[1-x^{k n}\left(\frac{1-x^{(2 d-2) k n}}{1+x^{k n}}\right)-x^{(2 d-1) k n}\right] \\
& =\prod_{n \in B}\left(\frac{1}{1-x^{n}}\right)\left(\frac{1}{1+x^{k n}}\right)\left[\left(1+x^{k n}\right)-x^{k n}\left(1-x^{(2 d-2) k n}\right)\right. \\
& =\prod_{n \in B}\left(\frac{1}{1-x^{n}}\right)\left(\frac{1}{1+x^{k n}}\right)\left[1-x^{2 d k n}\right] \\
& =\prod_{n \in B}\left(\frac{1}{1-x^{n}}\right)\left(\frac{1-x^{k n}}{1-x^{2 k n}}\right)\left(1-x^{2 d k n}\right) .
\end{aligned}
$$

Now $k B \cup 2 d k B \subseteq B \cup 2 k B$. So terms of the forms $1-x^{k n}$ and $1-x^{2 d k n}$ with $n \in B$, cancel with terms of the form $1-x^{m}$ or $1-x^{2 k m}$ with $m \in B$. In fact, $k B \cap 2 d k B \subseteq B \cap 2 k B$. So, if for some $n, n^{\prime} \in B$, $1-x^{k n}=1-x^{2 d k n^{\prime}}$, then both terms cancel with terms $1-x^{m}=1-x^{2 k m}{ }^{\prime}$ with $m, m^{\prime} \in B$. Also, $B \cap 2 k B \subseteq k B \cup 2 d k B$. So, if $1-x^{n}=1-x^{2 k n^{\prime}}$ with $n, n^{\prime} \in B$, then at least one of these terms cancels with a term of the form $1-x^{k m}$ or $1-x^{2 d k m}$ with $m \in B$. Hence, this is the generating function for $S(n)$ for some $S$. 
The elements of $N$ which are in $S$ are those in $(B \cup 2 k B)-(k B \cup 2 d k B)$ or $(B \cap 2 k B)-(k B \cap 2 d k B)$.

Proof of Corollary 1. When $d=1,2 d k B=2 k B$, and the restriction on $B$ in Theorem 1 is

$$
k B \cap 2 k B \subseteq B \cap 2 k B \subseteq k B \cup 2 k B \subseteq B \cup 2 k B
$$

which is clearly satisfied if $k B \subseteq B$.

When $d=1$, there is no $i$ such that $1 \leq i \leq d-1$, so parts can appear at most $(2 d-1) k-1=k-1$ times.

Also,

$$
\begin{aligned}
S & =[(B \cup 2 k B)-(k B \cup 2 k B)] \cup[(B \cap 2 k B)-(k B \cap 2 k B)] \\
& =[(B-k B)-((B-k B) \cap 2 k B)] \cup[(B-k B) \cap 2 k B] \\
& =B-k B .
\end{aligned}
$$

Proof of Corollary 2. When $k=1, k B=B$ and $2 d k B=2 d B$. So, the restriction on $B$ in Theorem 1 is

$$
B \cap 2 d B \subseteq B \cap 2 B \subseteq B \cup 2 d B \subseteq B \cup 2 B
$$

which is clearly satisfied if $2 d B \subseteq 2 B$; i.e., $d B \subseteq B$.

When $k=1$, no part can appear $2 i-1$ times with $1 \leq i \leq d-1$ or more than $2 d-2$ times. So parts can appear $2 i$ times with $1 \leq i \leq d-1$.

Also,

$$
\begin{aligned}
S & =[(B \cup 2 B)-(B \cup 2 d B)] \cup[(B \cap 2 B)-(B \cap 2 d B)] \\
& =[(2 B-2 d B)-((2 B-2 d B) \cap B)] \cup[(2 B-2 d B) \cap B] \\
& =2 B-2 d B=2[B-d B] .
\end{aligned}
$$

Proof of Corollary 3. When $B=\left\{i d^{j} \mid j \geq 0,1 \leq i \leq d-1\right\}, d B=\left\{i d^{j} \mid j \geq\right.$ $1,1 \leq i \leq d-1\} \subseteq B$.

Also,

$$
\begin{aligned}
S & =2[B-d B]=2\left[\left\{i d^{j} \mid j=0,1 \leq i \leq d-1\right\}\right] \\
& =\{2 i \mid 1 \leq i \leq d-1\}=A .
\end{aligned}
$$

Proof of Theorem 2. The generating function for $A^{*}(n, B)$ is

$$
\begin{aligned}
\prod_{n \in B}\left[\frac{1}{1-x^{n}}\right. & \left.-\sum_{i=1}^{d} \sum_{j=0}^{k-1} x^{((2 i-1) k+j) n}\right] \\
& =\prod_{n \in B}\left[\frac{1}{1-x^{n}}-\left(\sum_{i=1}^{d} x^{(2 i-1) k n}\right)\left(\sum_{j=0}^{k-1} x^{j n}\right)\right]
\end{aligned}
$$




$$
\begin{aligned}
& =\prod_{n \in B}\left[\frac{1}{1-x^{n}}-x^{k n}\left(\frac{1-x^{2 d k n}}{1-x^{2 k n}}\right)\left(\frac{1-x^{k n}}{1-x^{n}}\right)\right] \\
& =\prod_{n \in B}\left(\frac{1}{1-x^{n}}\right)\left[1-x^{k n}\left(\frac{1-x^{2 d k n}}{1+x^{k n}}\right)\right] \\
& =\prod_{n \in B}\left(\frac{1}{1-x^{n}}\right)\left(\frac{1}{1+x^{k n}}\right)\left[\left(1+x^{k n}\right)-x^{k n}\left(1-x^{2 d k n}\right)\right] \\
& =\prod_{n \in B}\left(\frac{1}{1-x^{n}}\right)\left(\frac{1}{1+x^{k n}}\right)\left[1+x^{(2 d+1) k n}\right] \\
& =\prod_{n \in B}\left(\frac{1}{1-x^{n}}\right)\left(\frac{1-x^{k n}}{1-x^{2 k n}}\right)\left(\frac{1-x^{(4 d+2) k n}}{1-x^{(2 d+1) k n}}\right) .
\end{aligned}
$$

Now $k B \cup(4 d+2) k B \subseteq B \cup 2 k B \cup(2 d+1) k B$. So terms of the forms $1-x^{k n}$ and $1-x^{(4 d+2) k n}$, with $n \in B$, cancel with terms of the form 1 $x^{m}$ or $1-x^{2 k m}$ or $1-x^{(2 d+1) k m}$ with $m \in B$. In fact, $k B \cap(4 d+2) k B \subseteq(B \cap 2 k B) \cup(B \cap(2 d+1) k B) \cup(2 k B \cap(2 d+1) k B)$. So, if $1-x^{k n}=1-x^{(4 d+2) k n^{\prime}}$ with $n, n^{\prime} \in B$, then both terms cancel with terms $1-x^{m}=1-x^{2 k m^{\prime}}$ or $1-x^{m}=1-x^{(2 d+1) k m}$ or $1-x^{2 k m}=$ $1-x^{(2 d+1) k m}$ with $m, m^{\prime} \in B$.

$(B \cap 2 k B) \cup(B \cap(2 d+1) k B) \cup(2 k B \cap(2 d+1) k B) \subseteq k B \cup(4 d+2) k B$.

So, if $1-x^{n}=1-x^{2 k n^{\prime}}$ or $1-x^{n}=1-x^{(2 d+1) k n^{\prime}}$ or $1-x^{2 k n}$ $=1-x^{(2 d+1) k n^{\prime}}$ with $n, n^{\prime} \in B$, then at least one of these terms cancels with a term of the form $1-x^{k m}$ or $1-x^{(4 d+2) k m}$ with $m \in B$.

Additionally, $B \cap 2 k B \cap(2 d+1) k B \subseteq k B \cap(4 d+2) k B$. So, if $1-x^{n}$ $=1-x^{2 k n^{\prime}}=1-x^{(2 d+1) k n^{\prime \prime}}$ with $n, n^{\prime}, n^{\prime \prime}, \in B$, then two of these terms cancel with terms $1-x^{k m}=1-x^{(4 d+2) k m}$ with $m, m^{\prime} \in B$. Hence, this is the generating function for $S(n)$ for some $S$.

The elements of $N$ which are in $S$ are those in

$$
(B \cup 2 k B \cup(2 d+1) k B)-(k B \cup(4 d+2) k B)
$$

or in

$[(B \cap 2 k B) \cup(B \cap(2 d+1) k B) \cup(2 k B \cap(2 d+1) k B)]-[k B \cap(4 d+2) k B]$ or in $B \cap 2 k B \cap(2 d+1) k B$.

Proof of Corollary 4. When $k=1$ and $B=\left\{2^{i} \mid i \geq u-1\right\}, k B=B, 2 k B$ $=2 B=\left\{2^{i} \mid i \geq u\right\},(2 d+1) k B=(2 d+1) B=\left\{(2 d+1) 2^{i} \mid i \geq u-1\right\}$, and $(4 d+2) k B=(4 d+2) B=\left\{(2 d+1) 2^{i} \mid i \geq u\right\}$. Thus, $B \cap(2 d+1) k B=\varnothing, 2 k B \cap$ $(2 d+1) k B=\varnothing, k B \cap(4 d+2) k B=\varnothing$, and $2 k B \subseteq B$. Hence, the condition 
imposed on $B$ in Theorem 2 is

$$
\varnothing \subseteq \varnothing \subseteq 2 B \subseteq B \cup(4 d+2) B \subseteq B \cup(2 d+1) B,
$$

which is clearly satisfied.

Also,

$$
\begin{aligned}
S= & {[(B \cup 2 k B \cup(2 d+1) k B)-(k B \cup(4 d+2) k B)] } \\
& \cup[((B \cap 2 k B) \cup(B \cap(2 d+1) k B) \\
& \cup(2 k B \cap(2 d+1) k B))-(k B \cap(4 d+2) k B)] \\
& \cup[B \cap 2 k B \cap(2 d+1) k B] \\
= & {[(B \cup(2 d+1) B)-(B \cup(4 d+2) B)] \cup[2 B-\varnothing] \cup[\varnothing] } \\
= & {[(2 d+1) B-(4 d+2) B] \cup 2 B } \\
= & \left\{(2 d+1) 2^{i} \mid i=u-1\right\} \cup\left\{2^{i} \mid i \geq u\right\} .
\end{aligned}
$$

Proof of Theorem 3. The generating function for $A^{*}(n, B)$ is

$$
\begin{aligned}
\prod_{n \in B}\left[\frac{1}{1-x^{n}}-\right. & \left.\sum_{i=1}^{\infty} \sum_{j=0}^{k-1} x^{((2 i-1) k+j) n}\right] \\
& =\prod_{n \in B}\left[\frac{1}{1-x^{n}}-\left(\sum_{i=1}^{\infty} x^{(2 i-1) k n}\right)\left(\sum_{j=0}^{k-1} x^{j n}\right)\right] \\
& =\prod_{n \in B}\left[\frac{1}{1-x^{n}}-x^{k n}\left(\frac{1}{1-x^{2 k n}}\right)\left(\frac{1-x^{k n}}{1-x^{n}}\right)\right] \\
& =\prod_{n \in B}\left(\frac{1}{1-x^{n}}\right)\left[1-x^{k n}\left(\frac{1}{1+x^{k n}}\right)\right] \\
& =\prod_{n \in B}\left(\frac{1}{1-x^{n}}\right)\left(\frac{1}{1+x^{k n}}\right)=\prod_{n \in B}\left(\frac{1}{1-x^{n}}\right)\left(\frac{1-x^{k n}}{1-x^{2 k n}}\right) .
\end{aligned}
$$

Now, $k B \subseteq B \cup 2 k B$. So, terms of the form $1-x^{k n}$ with $n \in B$, cancel with terms of the form $1-x^{m}$ or $1-x^{2 k m}$ with $m \in B$. Also, $B \cap 2 k B \subseteq$ $k B$. So, if $1-x^{n}=1-x^{2 k n^{\prime}}$ with $n, n^{\prime} \in B$, then one of these terms cancels with a term of the form $1-x^{k m}$ with $m \in B$. Hence, this is the generating function for $S(n)$ for some $S$.

The elements of $N$ which are in $S$ are those in $(B \cup 2 k B)-k B$ or in $B \cap 2 k B$.

\section{REFERENCES}

1. H. L. Alder, Partition identities-from Euler to the present, Amer. Math。 Monthly 76 (1969), 733-746. MR 41 \#8366. 
2. G. E. Andrews, Number theory, Saunders, Philadelphia, Pa., 1971. MR $46 \# 8943$.

3. - Two theorems of Euler and a general partition theorem, Proc. Amer. Math. Soc. 20 (1969), 499-502. MR 38 \#2112.

4. J. W. L. Glaisher, Messenger Math. 12 (1883), 158-170.

5. G. Lord, Ph.D. Thesis, Temple University, March 1971.

6. E. Moore, Generalized Euler-type partition identities, J. Combinatorial Theory Ser. A 17 (1974), 78-83.

7. - Partitions with parts appearing a specified number of times, Proc. Amer. Math. Soc. 46 (1974), 205-210.

8. M. V. Subbarao, Partition theorems for Euler pairs, Proc. Amer. Math. Soc. 28 (1971), 330-336. MR 43 \#175.

DEPARTMENT OF MATHEMATICS, HUMBOLDT STATE UNIVERSITY, ARCATA, CALIFORNIA 95521 